\title{
Das Kastensystem der Jaunsari (Nordindien)
}

Susanne Haas

Die Jaunsari gehören zu den nichttibetischen, als Pahari oder Bergbewohner bezeichneten Bevölkerungen der unteren Himalaja-Ränge, zwischen dem südöstlichen Kaschmir und Nepal. Ihr Wohngebiet ist Jaunsar-Bawar, der gebirgige Teil des zu Uttar Pradesh gehörenden Distriktes Dehra Dun. Sie sprechen eine arische Sprache, den jaunsarischen Dialekt des West-Pahari. Ihre Kultur weist viele eigenartige Züge auf, ist aber letztlich als hinduistisch zu bezeichnen.

Ich sammelte das vorliegende Material in den Jahren 1958 und 1959, nachdem mir die indische Regierung, auf Empfehlung meines verehrten Lehrers Herrn Prof. Dr. Alfred Steinmann hin, ein Austauschstipendium gewährt hatte. Ich arbeitete westiich des Hauptortes Chakrata, vor allem in den Dörfern Dasau und Haja. Die übrigen Dörfer, die ich erwähnen werde, befinden sich im selben Gebiet.

\section{Die jaunsarischen Kasten im Überblick}

Der hinduistische Kastengedanke ist auch bei den Jaunsari lebendig: die jaunsarische Gesellschaft setzt sich aus einer Folge von geburtlich fixierten, religiös verankerten und wirtschaftlich spezialisierten Schichten zusammen.

An der Spitze der jaunsarischen Kastenhierarchie stehen die Brahmanen. Ein Teil von ihnen, die «echten» Brahmanen (asli oder khas Brahman), aus deren Reihen sich die Priester rekrutieren, halten streng auf Kastenendogamie, gehen also nur untereinander oder mit andern Brahmanen Ehen ein. Ein anderer Teil, die "vermischten» Brahmanen (Brahmshankara), die von Ackerbau und Viehzucht leben, dulden dagegen Zwischenheiraten mit der nächsten Kaste, den Rajput. «Echte» Brahmanen lernte ich den Dörfern Mathiyana und Toda kennen, "vermischte» in Haja und Jagthan.

Die Rajput, die die zweithöchste Stelle in der Kastenhierarchie einnehmen, betrachten sich als Kshatriya, das heißt Angehörige der klassischen Herrscher- und Kriegerkaste. Sie bezeichnen sich auch als Khosh (meist Khasa geschrieben). Es ist wahrscheinlich, daß sie, zusammen mit den Khasiya von Garhwal und Kumaon und weiteren $\mathrm{Pa}$ hari-Rajput, von den Khasa abstammen, die im sanskritischen Schrifttum mehrfach erwähnt und im Gesetzbuch des Manu (Manu Smriti X: 43, 44) als eine wegen Mißachtung religiöser Vorschriften degradierte Kshatriya-Gruppe bezeichnet worden sind. Zur Zeit der mohammedanischen Herrschaft mögen Rajput aus dem Tiefland zu ihnen gestoßen sein (vgl. Saksena 1955: $10 \mathrm{ff}$.).

Wie die "vermischten" Brahmanen und auch manche unter den "echten" betreiben die jaunsarischen Rajput Ackerbau und Viehzucht.

Auf die genannten hohen Kasten (bade Jat) folgen die Handwerker, die Musikanten und einige weitere professionelle Kasten. Sie werden von den Jaunsari schon als niedrig (kamin) klassifiziert, stehen aber in der Praxis zwischen den hohen Kasten und der niedrigsten sozialen Schicht. Wie Majumdar (1962: 23) bezeichne ich sie deshalb als mittlere Kasten. Unter ihnen besitzen die Badhi oder Zimmerleute eindeutig den höchsten Status. Zwischen den andern mir bekannten Kasten dieser Kategorie, den Sonar oder Goldschmieden, den Lohar oder Eisenschmieden und den Bajgi oder Musikanten, herrscht etwelche Statusrivalität; von Leuten hoher Kaste werden sie aber ungefähr in der erwähnten Reihenfolge eingestuft. Ebenfalls zu den mittleren Kasten gehören die Jogra und die Nath, die sich als Pilgerführer, Zauberer und Astrologen betätigen (vgl. Majumdar 1962: 23 und 67); ich begegnete diesen nur spärlich vertretenen Kasten im Gebiet von Dasau und Haja nicht.

Auf der untersten Stufe der sozialen Hierarchie befinden sich die Kolta ${ }^{1}$, die auch etwa als Koi, Koli, Chamar (im Tiefland die Kaste der Abdecker und Lederarbeiter) oder Dom bezeichnet werden. Ihre Geschichte liegt noch im dunklen, aber es ist denkbar, daß sie die Nachkommen einer einheimischen Bevölkerung sind, die von arisch sprechenden Einwanderen unterjocht worden war (vgl. Saksena 1955: 14 und Majumdar 1962: vii). Kolta erinnert übrigens an Kuluta, den Namen eines alten nordindischen Volkes, das, wie die Khasa, in der Brihat Samhita (14: 22 und 29) genannt ist.

Das Wort Dom wird gelegentlich auch als Sammelname für alle unterhalb der Rajput stehenden Kasten verwendet. Viele Leute lehnen diesen verallgemeinernden Gebrauch aber angesichts des Statusgefälles zwischen den mittleren Kasten und den Kolta ab. Möglicherweise stammen die mittleren Kasten aus der gleichen ethnischen Einheit wie die Kolta; denn berufliche Spezialisierung kann zur Bildung neuer Kasten führen. 
In Haja fand ich, daß die Brahmanen von ihren Dorfgenossen mittlerer Kaste mit dem Ausdruck "unsere Punde» und von ihren Kolta mit "unsere Bohra» bezeichnet werden. Mit dem Possessivpronomen wird auf die wirtschaftlichen Beziehungen hingewiesen, die zwischen den verschiedenen Kasten eines Dorfes bestehen; von ihnen wird später ausführlich die Rede sein. Zu Bohra ist zu bemerken, $\mathrm{da} ß$ es in Kaschmir eine hinduistische, den brahmanischen Pandit nahestehende Minorität gleichen Namens gibt (vgl. Madan 1965: 16); vielleicht besteht ein Zusammenhang. Die «echten» Brahmanen werden nicht als Bohra bezeichnet.

Für die zu häuslichen Kulten beigezogenen Brahmanen verwenden die Leute die Bezeichnung «unsere Purohit (Hauspriester)», und diese nehmen auf ihre Kunden mit dem Ausdruck «unsere Jajman (Opferherren)» Bezug. Tempelpriester werden dagegen als Pujari oder Pujgi (von sanskrit puj- verehren) bezeichnet.

Die Rajput sind für die Badhi ihres Dorfes «unsere Deshtiya», für die Bajgi «unsere Negi (Empfänger von Neg, d. h. Gratifikationen, u. a. ein Beamtentitel)» und für die Kolta « unsereThakur (von sanskrit Thakkura Gottheit, Gegenstand der Verehrung)» oder «unsere Mosh (evtl. abzuleiten von sanskrit mahashaya hochgesinnt, edel)». Zu Mosh ist $\mathrm{zu}$ sagen, daß in der Gegend von Dasau zwei Arten von Rajput unterschieden werden: Mosh und Dui (u nasaliert). Mosh stellte ich außer in Dasau auch in Sunora und Kwanu fest, Dui in Jadi und Loharni. Zwischen diesen Gruppen herrscht einige Rivalität: jede hält sich für höherstehend. Zum Bissu-Fest, das jeweils zu Beginn des Monats Baisakh (Mitte April) veranstaltet wird und in dessen Mittelpunkt Duelle mit Bogen und Pfeil stehen, erscheinen in Dasau auch Delegationen aus der Nachbarschaft. Kämpfe zwischen Mosh und Dui stoßen dabei immer auf besonderes Interesse. Heiraten zwischen Mosh und Dui kommen vor, den Ehepartner innerhalb der eigenen Gruppe zu wählen gilt aber als feiner. Der Dorfobmann von Dasau identifizierte die Mosh mit den Chauhan, die Dui mit den Tomar, zwei im Tiefland verbreiteten Rajput-Klans. Diese Theorie schien aber seinem eigenen Kopf entsprungen zu sein, denn meinen andern Informanten war sie nicht bekannt.

Für die Badhi gibt es kein besonderes Bezugswort. Dagegen werden die in einem festen Dienstverhältnis zu ihren Dorfgenossen hoher Kaste stehenden Bajgi von diesen mit «unsere Dhaki (Trommler)» bezeichnet und damit von den Dewar, den als Tempeldiener für eine Gottheit tätigen Bajgi, unterschieden.

Die Kolta werden von ihren Dienstherren mit dem Ausdruck «unsere $\mathrm{Hali}^{2}$ » bezeichnet.

Allein oder zusammen mit persönlichen Namen dienen die Termini Punde, Bohra, Deshtiya, Negi,
Thakur, Mosh und Dui auch als Anrede. Brahmanen und Rajput sprechen sich mit dem persönlichen Namen an, dem sie manchmal noch das höfliche Mama (Muttersbruder) beifügen. Leute niedrigerer Kaste werden mit Badhi, Dhaki, Dewar, Koi oder Dom oder einfach mit dem persönlichen Namen angesprochen.

Majumdar (1962: vii und 24) stellte fest, daß die jaunsarischen Brahmanen und Rajput, wie ihre Kastengenossen im Tiefland, die rassischen Züge aufweisen, die etwa als «indo-arisch» oder «mediterran» bezeichnet werden. Bei den niedrigeren Kasten glaubte er gelegentlich auch andere Züge gefunden zu haben (er verwendet in diesem Zusammenhang die zum Teil aus der Sprachforschung stammenden Bezeichnungen "praedrawidisch", «austrisch» und "protoaustralid»). An eingehenden anthropologischen Untersuchungen fehlt es noch.

Über die zahlenmäßige Stärke der einzelnen Kasten liegen keine genauen Angaben vor. Am besten vertreten sind aber zweifellos die Rajput, am schwächsten die mittleren Kasten (vgl. Majumdar 1962: 22 ff.). Ich zählte in Dasau 23 Rajput-, 2 Badhi-, 4 Bajgi- und 7 Kolta-Haushalte und in Haja 32 Brahmanen-, 5 Badhi-, 5 Bajgi-, 2 Sonar- und 14 Kolta-Haushalte.

In den meisten Dörfern leben Rajput oder Brahmanen und daneben Handwerker, Bajgi und Kolta. Mir ist kein Dorf bekannt, in dem Brahmanen und Rajput in größerer Zahl nebeneinander lebten.

Wie schon Majumdar (1962: 24 und $40 \mathrm{ff}$.) feststellte, erklären die Brahmanen, die Rajput und gelegentlich auch die Angehörigen der übrigen, im allgemeinen weniger traditionsbewußten Kasten, von Sirmur, Jubbal, Tehri Garhwal oder sogar Kaschmir her nach Jaunsar-Bawar gekommen zu sein. In Dasau erzählten mir meine Informanten, ihr Dorf sei ursprünglich nur von Kolta besiedelt gewesen. Deshalb werde es heute noch inoffiziell Koiya (von $K o i=$ Kolta) genannt. Die Vorfahren der Rajput seien vor einigen Jahrhunderten von Koti in Sirmur und Ogalta in Jubbal her eingewandert. Einer der Einwanderer habe Dashu geheißen, daher der Name Dasau. Mit Koti unterhielten die Rajput übrigens freundschaftliche Beziehungen: 1957 hatten Männer aus Koti am Mand-Fest von Dasau, das im Monat Asarh (Mai-Juni) abgehalten wird und im wesentlichen aus einer Fischfangexpedition an den Tons-Fluß besteht, teilgenommen, und 1958 hatte eine Delegation aus Dasau den Besuch erwidert. Die Brahmanen von Haja erzählten, sie stammten von einem Priester aus Kaschmir ab, der in Himachal Pradesh im Dienste eines Rout (von Rawat, Herrscher) gestanden sei. Eines Tages habe der Priester seinen Herrn erschlagen, mit dessen Frau die Flucht ergriffen und sich schließlich in Haja niedergelassen. 
Meine Auskunftgeber betrachteten das Kastenwesen als Teil der göttlichen Weltordnung. Der Dorfobmann von Dasau erklärte mir: "Auch die Kolta sind Menschen, aber Gott hat sie zum Dienen geschaffen.» Und selbst diejenigen unter den Kolta, die unter dem Einfluß von Sozialarbeitern und Beamten begonnen hatten, die Vorrechte der hohen Kasten in Frage zu stellen, hätten sich aus Furcht vor dem Zorn der Götter nie in die ihrer Kaste verbotene Tempelzone begeben.

Úberzeugt davon, die Götter auf ihrer Seite zu haben, sprachen die Rajput und die Brahmanen den übrigen Kasten das Recht, Land zu erwerben, ab. Bis in die neueste Zeit befand sich deshalb alles Land im Besitze der Familien hoher Kaste. Rajput, mit denen ich über diese Verhältnisse sprach, zitierten mir die folgende Strophe aus einem Lied, das sie bei kultischen Festlichkeiten zu singen pflegten (ich stütze mich auf die Hindi-Übersetzung, die mir ein Rajput gab):

Der Gottheit wird eine Ziege gegeben (als Opfer) Der Bajgi erhält das Haupt (als Opferlohn)

Der Rajput pflügt die Felder

Der Kolta verlor, als er drinnen pflügte

Die Erklärung zu dieser Strophe lautete:

"Es waren einmal zwei Brüder, der ältere ein Rajput, der jüngere ein Kolta. Beide begehrten das Land für sich. Sie beschlossen, ein Wettpflügen zu veranstalten: wieviel jeder zu pflügen vermöchte, sollte er behalten dürfen. Der ältere brachte der Gottheit eine Ziege dar, führte seine Ochsen aufs Feld, spannte sie an und begann zu pflügen. Der jüngere unterlie $\beta$ das Opfer, spannte die Ochsen aus Torheit schon im Stall an und blieb dann mit ihnen in der Türe stecken. So zeigte Gott, daß er den Rajput zum Landbesitzer, den Kolta zum Knecht bestimmt hatte.»

Wie die Wirtschaft, so wird auch das politische Leben von den hohen Kasten beherrscht. Sie stellen den Dorfobmann, und sie bildeten auch bis vor kurzem den Dorfrat (in den letzten Jahren gestalteten die Behörden die Räte um).

Ein weiteres Privileg, hinter dem ich übrigens einen Astralmythos vermute, betrifft das Tragen von Schmuck: Goldschmuck ist den hohen und den mittleren Kasten vorbehalten; die Kolta haben sich mit Silber zu begnügen.

Im täglichen Verkehr haben die niedrigen Kasten die höheren als erste zu grüßen und ihnen ehrerbietig den Weg freizugeben.

Die hinduistische Lehre von der Wiedergeburt, laut welcher der Mensch nach dem Tod seinen Verdiensten gemäß in einer hohen oder einer niederen Kaste

wiedergeboren wird, beschäftigte meine Informanten kaum. Wie bei den Hindu des Tieflandes spielte aber auch bei ihnen der Begriff einer Art von Reinheit eine große Rolle. Meine Auskunftgeber unterschieden "reine» und "unberührbare» Kasten (suddha und achut Jat) und innerhalb dieser Kategorien wiederum Kasten von größerer und solche von geringerer "Reinheit». "Unberührbar» bedeutete dabei so viel wie "unrein»; der Ausdruck hängt mit der Vorstellung zusammen, "Unreinheit» könne durch Kontakte übertragen werden. Brahmanen und Rajput bilden die Kategorie der «reinen» Kasten, die übrigen Leute die der «unberührbaren». Innerhalb ihrer Kategorie gelten Brahmanen als «reiner» denn Rajput, Badhi als «reiner» denn Sonar, Lohar und Bajgi und diese wiederum als "reiner» denn Kolta.

Der beschriebene Reinheitskomplex ist im religiösrituellen Bereich verankert: «Reinheit» ist Voraussetzung zum Verkehr mit dem Heiligen, Göttlichen und damit zur Zugehörigkeit zu den Opferpriestern und den Opferern der jaunsarischen Hauptgottheiten. Nur die Inhaber des höchsten «Reinheitsgrades», die Brahmanen, können an einem MahasuTempel Priester werden, weil sie allein es wagen dürfen, den Hauptraum, in dem sich die Idole befinden, zu betreten; die Rajput haben dagegen nur bis zum Vorraum Zutritt, die Handwerker und die Bajgi müssen sich mit der Tempelplattform begnügen, und die Kolta haben auch die Plattform zu meiden. Diese Vorschriften wurden genau eingehalten, denn jedermann glaubte, die Gottheiten würden Zuwiderhandelnde töten oder sonstwie mit Unglück schlagen. Als ich in Dasau einmal im Vorraum des Tempels von Chalta Mahasu mit Blitzlicht fotographierte, entstand gleich das Gerücht, ich sei von niedriger Kaste, denn die Gottheit habe mich mit einem Blitzstrahl gegen eine Wand geworfen.

Kinder werden bei den Jaunsari in die Kaste ihrer Väter geboren. Die Kastenmitgliedschaft kann aber 
im Laufe des Lebens verwirkt werden. Diese Gefahr droht den einzelnen Mitgliedern, wenn sie sich «verunreinigen». Eine "Verunreinigung» kann aus dem Genuß bestimmter Nahrungsmittel oder Getränke oder aus direkten oder indirekten Kontakten mit Leuten niedrigerer, also «weniger reiner» Kaste resultieren. Mit dieser Vorstellung hängen zahlreiche Meidungsvorschriften zusammen. Sie betreffen

1. den Genuß von Fleisch: Brahmanen dürfen an Fleisch von Haustieren nur Ziegenbock und Ziege, Rajput nur Ziegenbock, Ziege und Widder, Angehörige der mittleren Kasten nur Ziegenbock, Ziege, Widder, Schaf und Huhn essen. Einzig den Kolta ist der Genuß des Fleisches von Schwein und Rind, einschließlich Rindkadavern, erlaubt; aber an einigen Orten, u. a. in Dasau, lehnen seit einigen Jahren auch die Kolta Rindfleisch ab. Die Brahmanen und die Rajput bringen die sie betreffenden Verbote damit in Zusammenhang, daß ihre Hauptgottheiten von ihnen nur Ziegenböcke, bzw. Ziegenböcke und Widder, als Opfer annehmen. In bezug auf Widder als Opfer ist zu sagen, daß dieses Tier bei den alten Indern die Zeugungskraft verkörperte und daß geschnitzte Widderköpfe in der jaunsarischen Hausornamentik, die zweifellos symbolträchtig ist, eine Rolle spielen. Das Fleisch von Schafen und Rindern wird mit dem Argument, diese Tiere seien "Mütter», das heißt Verkörperungen der weiblichen Fruchtbarkeit, abgelehnt; dagegen drückt man bei Ziegen ein Auge zu. Schweine und Hühner gelten als "unrein», und zwar angeblich, weil sie sich von Unrat ernährten; aber vermutlich hängt die Abscheu mit alten, von den Mahasu-Verehrern nicht gebilligten Opferbräuchen zusammen. - Eier werden wie Hühner behandelt. - Der Genuß von Wild, vor allem von Cerviden und Wildhuhn, ist dagegen gestattet, und auch Fisch darf von allen Kasten gegessen werden.

2. den Genuß von Alkohol: Brahmanen haben sich alkoholischer Getränke zu enthalten. Auch dieses Verbot dürfte letztlich mit alten Opferbräuchen, bei denen Alkohol eine Rolle spielte, zusammenhängen.

\section{3. die Wahl der Partner für Ehe und Liebschaften:} «Echte» Brahmanen dürfen zwar mit «vermischten», nicht aber mit Rajput Ehen eingehen. Eheschließungen zwischen "vermischten» Brahmanen und Rajput wie auch solche zwischen Angehörigen der mittleren Kasten sind erlaubt, und zwar ist sowohl Hyper- als auch Hypogamie möglich: in Dasau fand ich, daß von den 70 Gattinnen von Rajput 5 der Brahmanen-Kaste angehörten, und in Haja gab es mehrerer Rajput-Frauen, die mit Brahmanen verheiratet waren. Heiraten zwischen den übrigen Kasten sind verboten. - Außereheliche Beziehun- gen spielen im Leben der Jaunsari eine große Rolle. Den hohen Kasten ist es aber nicht gestattet, sich mit Kolta einzulassen, und auch Beziehungen zu Handwerkern oder Bajgi gelten als unkorrekt. Die mittleren Kasten haben sich des Verkehrs mit Kolta zu enthalten.

\section{Einladungen zum Essen, Trinken und Rauchen:} Brahmanen und Rajput dürfen voneinander, nicht aber von den übrigen Kasten Nahrung, Wasser und die Wasserpfeife annehmen. Es ist ihnen aber erlaubt, ihre eigene metallene Wasserpfeife nach Entfernen des Saugrohres und ihre Bambuspfeife, die sie jeweils ins Feld mitnehmen, samt Saugrohr den mittleren Kasten anzubieten. Auch dürfen sie den Pfeifenkopf der Bambuspfeife den Kolta reichen. - Brahmanen sollten von Rajput benütztes Eßoder Trinkgeschirr nicht eigenhändig reinigen; deshalb müssen Rajput, die im Hause von Brahmanen eingeladen sind, ihr Geschirr selber waschen (in der Praxis genügt es, wenn sie ein wenig Wasser hineingießen). Brahmanen sollten Rajput-Gästen die Füße nicht eigenhändig waschen (gleichrangigen Gästen muß eine Waschung geboten werden), sondern nur warmes Wasser bereitstellen. Handwerkern und Bajgi ist es erlaubt, voneinander, nicht aber von Kolta Nahrung, Wasser und Rauchzeug anzunehmen.

5. das Betreten der Wohnhäuser: Brahmanen und Rajput dürfen Angehörige der mittleren Kasten nur bis in die Mitte des Wohnraumes, in dessen hinterem Teil sich die Herdstelle befindet, einlassen und Kolta nur bis in den Vorraum, weil sonst der Herd und damit das ganze Haus «unrein» würde. Desgleichen dürfen die mittleren Kasten Kolta höchstens bis in den Vorraum kommen lassen. - In den Häusern von Leuten mittlerer Kaste mögen sich Brahmanen und Rajput nach Belieben aufhalten (wenn die Bajgi auf Festtage hin noch nachts Licht haben, weil sie für ihre Kunden Festkleider nähen müssen, trifft man sich oft bei ihnen zu einem Schwatz); dagegen sollten sie in Kolta-Häusern nicht längere Zeit verweilen.

6. die Zuweisung von Wohnquartieren: Die hohen Kasten haben dafür zu sorgen, daß sich in ihrem Wohnbereich keine Kolta niederlassen. Die Kolta sind in tiefer gelegene Zonen zu verweisen.

7. die Benützung von Brunnen: Die hohen Kasten haben dafür zu sorgen, daß ihre Brunnen nicht von Kolta benützt werden. Die Kolta dürfen nur in einer tiefer gelegenen Zone Wasser schöpfen.

Ist ein Verstoß gegen ein solches Verbot ruchbar geworden, so sollte der Fehlbare von seinen Kastengenossen im Rahmen einer ad hoc zusammenge- 
rufenen und vom Kastenältesten geleiteten Versammlung zur Rechenschaft gezogen werden. Erweist er sich als uneinsichtig, so sollte er aus der Kaste ausgestoßen, das heißt fortan gemieden werden, damit seine "Unreinheit» nicht die Kaste infisziere. In der Praxis scheinen solche Ausstoßungen selten zu sein. Ein aus Dasau stammender Rajput, der im Tiefland eine Frau niederer Kaste geheiratet hatte, wurde aber einmal auf Grund stillschweigenden Übereinkommens geächtet; als er sein Heimatdorf besuchte, wurde ihm nur in seinem Elternhaus Einlaß gewährt.

Viele Verstöße bleiben meiner Erfahrung nach ungeahndet. In Dasau beobachtete ich, daß manche Rajput nicht zögerten, Handwerkern und Bajgi im kleinen Kreis die metallene Wasserpfeife samt Rohr anzubieten. Einmal hörte ich, wie ein Rajput-Kind seine Vatersschwester rügte, weil sie ihre Pfeife einer Bajgi-Frau gereicht hatte. Beide Frauen lachten aber nur darüber. Auch nahm man es in Dasau hin, daß einer der Rajput mit einer Kolta-Frau und einer der Kolta mit einer Rajput-Frau ein heimliches Verhältnis pflegte, und eine junge Kolta-Frau klagte mir einmal, daß ihr viele Rajput nachstellten. Ebenfalls in Dasau duldeten die Rajput einen Kolta in ihrem Wohnquartier, nachdem er versprochen hatte, keine Schweine mehr zu halten. In Haja hörte ich von der Tochter eines Brahmanen, die nach mehreren gescheiterten Ehen die Frau eines Bajgi geworden war. Und im gleichen Dorf fand ich, daß einige Brahmanen, darunter auch der Dorfobmann, im stillen Schnaps tranken.

Als Mittel zur Tilgung einer "Verunreinigung» wurden mir Kuhurin, Feuer und Ziegenopfer genannt. Wer "unrein" geworden ist, kann sich mit einem Trank aus Kuhurin, verdünnt mit Wasser, reinigen. Ist ein Haus "verunreinigt» worden, so muß der Besitzer den Herd mit Kuhurin waschen und auf der Schwelle zum Wohnraum eine Ziege opfern. Zu diesem Schritt sah sich einmal ein Brahmane von Haja genötigt, weil sein Haus von einem Distriktsbeamten niederer Kaste betreten worden war. Auch hörte ich, daß zu Beginn meines Feldaufenthaltes ein Dorfobmann, der über meine Kaste im Zweifel war, eine Ziege geopfert habe, nachdem er mich in seinem Haus empfangen hatte. Ist ein metallener Gegenstand «verunreinigt» worden, so hält man ihn ins Feuer.

\section{Die wirtschaftliche Verkettung}

Die jaunsarische Wirtschaft wird weitgehend von den Landwirten kontrolliert. $\mathrm{Zu}$ ihnen stehen sowohl die Priester als auch die Handwerker, die Bajgi und die Kolta in einem mehr oder weniger engen Dienstverhältnis.
Die Priester rekrutieren sich aus der BrahmanenKaste, und zwar wie erwähnt aus der Kategorie der "echten» Brahmanen. Einige von ihnen sind auf den Dienst an den Tempeln der großen Gottheiten spezialisiert: sie versehen das Priesteramt jedes Jahr während einiger Monate, leben während dieser Zeit getrennt von ihrer Familie, also sozusagen im Zölibat, lassen sich aber dann wieder ablösen. Die andern betätigen sich als Haus- und vereinzelt auch als Dorfpriester: als Hauspriester stehen sie im Dienst einer ererbten oder im Laufe der Zeit erworbenen Kundschaft, für die sie alle bei Geburten, Hochzeiten, Todes- und Krankheitsfällen üblichen Zeremonien durchführen, als Dorfpriester leiten sie die zum Wohle der Gemeinde notwendigen Kulte. Sie leben mit ihrer Familie und betreiben meistens auch ein wenig Landwirtschaft. Für ihre Dienste erhalten sie nach jeder Ernte von ihren Rajput- und Brahmanen-Kunden und, falls sie auch als Dorfpriester tätig sind, von den übrigen Familien hoher Kaste einen kleinen Sack Getreide, nach allen Zeremonien einen Anteil an den Opfergaben und nach manchen eine der Dankbarkeit dies Kunden angemessene Gabe in Form von Geld, Schmuck, Vieh oder andern Gütern. Angehörige der mittleren Kasten revanchieren sich bei ihnen mit handwerklicher Arbeit oder mit Botengängen und Kolta mit Trägerdiensten. Zumal da die Kundschaft oft groß ist - ein mir bekannter Priester arbeitete für mehr als 40 Rajput- und Brahmanenfamilien - , sind die Priester wirtschaftlich fast durchwegs gut gestellt.

Die Kundschaft eines Hauspriesters ist ziemlich beständig; aber es steht den Kunden doch frei, auch andere Priester zu konsultieren. Ein Priester aus dem Norden von Jaunsar-Bawar, der einige Tage in Dasau weilte, um seine Frau von der Hebamme des Ortes behandeln zu lassen, wurde gleich von mehreren Rajput mit der Durchführung von Kulten gegen krankmachende Geister betraut. In Dasau fand ich auch, daß einige Rajput für kleinere Zeremonien den jungen Nachfolger ihres einstigen Hauspriesters holten, für gewichtigere aber einen renommierten Priester aus der weiteren Umgebung. Die Priester konkurrenzieren sich also gelegentlich.

Die Priester beziehen ihr Wissen aus einem auf Pahari und im Takari-Alphabet geschriebenen Buch, das den Namen "Kaschmirische Wissenschaft» (Kashmiri Vidhya) trägt. Dieses Buch, das angehende Priester bei ihrem Lehrer (dem Vater oder dem Muttersbruder) abschreiben, enthält Anweisungen zur Durchführung von Zeremonien, Opfersprüchen, magischen Diagrammen und Tabellen zum Bestimmen von Krankheiten und zum Erstellen von Horoskopen. Eine formelle Initiation der angehenden Priester soll nicht stattfinden. In diesem $\mathrm{Zu}$ sammenhang möchte ich bemerken, daß die jaunsarischen Brahmanen und Rajput, anders als ihre 
Brüder im Tiefland, die "heilige Schnur» nicht tragen und sich auch nicht als Zweimalgeborene bezeichnen.

Die Badhi betätigen sich als Zimmerleute, Schnitzer und Dreher, die Sonar als Goldschmiede und die Lohar als Eisenschmiede. Alle sind weitgehend selbständige Handwerker, die für jede Arbeit einen Lohn in Form von Geld oder Getreide verlangen. Als Anklang an ein festes Dienstverhältnis zwischen ihnen und den hohen Kasten mag man aber den Brauch betrachten, daß sie nach jeder Ernte bei den Landbesitzern ihres Dorfes einen kleinen Sack Getreide abholen dürfen. Auch würde man es ihnen sehr übelnehmen, wenn sie den Auftrag eines Dorfgenossen zurückwiesen.

Die Dewar unter den Bajgi betätigen sich als Tempelmusikanten und Tempeldiener. Die mir bekannten gehörten zum Gefolge der Gottheit Chalta Mahasu, deren Idol in Jaunsar-Bawar und im angrenzenden Gebiet von Himachal Pradesh von Dorf zu Dorf zirkuliert. Sie pflegen eine Geheimsprache'3, vermutlich ein Rotwelsch, die es ihnen ermöglicht, sich auch in Gegenwart von Opferern ungeniert über Opfergaben und Opferlöhne zu unterhalten.

Die als Dhaki bezeichneten Bajgi betätigen sich als Dorfmusikanten und dazu als Barbiere, Schneider, Boten und Opfergehilfen der Familien hoher Kaste. Dhaki-Frauen verrichten auch Hebammendienste. Jede Dhaki-Familie besitzt ihre eigene Kundschaft. In Dasau umfaßte diese 3-5 Rajput-Familien. Teilt sich eine Dhaki-Familie, so haben die aus dem alten Haushalt ausscheidenden Familienmitglieder Anrecht auf einen Teil der Kundschaft. Für ihre Dienste erhalten die Dhaki einen Sack Getreide von den Ernten ihrer Kunden, eine kleine Getreidegabe von den übrigen Dorfbewohnern hoher Kaste, eine Gabe, wenn in einem Kundenhaus zu einem Fest aufgespielt wurde, Bezahlung in Form von Geld oder Getreide für Schneiderarbeiten und die Köpfe der Opfertiere als Opferlohn.

Sowohl die Handwerker als auch die Bajgi verdienen normalerweise genug, um nicht nur das Leben fristen, sondern auch Schmuck und gute Kleider anschaffen zu können. Die Handwerker besitzen meist ein eigenes Haus; nur die Bajgi leben oft in einer von Kunden zur Verfügung gestellten Behausung. Manche Familien bewirtschaften auch ein wenig Pachtland oder Land, das ihnen von Kunden zur Nutzung überlassen wurde.

Die Badhi finden oft nicht genug Arbeit in ihrem Dorf und begeben sich deshalb während einiger Monate des Jahres auf die Wanderschaft. Desgleichen ziehen die jungen Bajgi manchmal aus, wenn ihre Väter ihrer Mithilfe noch nicht bedürfen.

Die Kolta verrichten für die hohen Kasten Knechtearbeit. Die meisten wohlhabenden Landbesitzer verfügen über eine Kolta-Familie. Sie überlassen dieser Familie eine einfache Behausung und ein
Stück Ackerland, gewähren ihr von Zeit zu Zeit ein Darlehen, strecken ihr den Brautpreis vor, wenn eines ihrer Mitglieder heiraten will, und verlangen dafür, daß die Männer und gelegentlich auch die Frauen für sie auf dem Feld arbeiten. Zur Erntezeit gewähren sie der Familie einen großen Sack Getreide je erwachsenes Mitglied und einen kleinen je Kind, und an den einzelnen Arbeitstagen bieten sie den Arbeitenden zwei Mahlzeiten. Vielfach dauert ein solches Dienstverhältnis Generationen. Die Kolta können sich nur an einen neuen Herrn verdingen, wenn dieser dem alten die Darlehen zurückzahlt, die sie im Lauf der Zeit aufgenommen hatten; denn durch ihre Arbeit vermögen sie zwar die Schuldzinsen, aber kaum je die Darlehen selbst zu kompensieren. Sie stecken also in Schuldknechtschaft. Indessen sollte man ob der Schuldenrechnung nicht übersehen, daß sie dem jaunsarischen Gefühl nach sozusagen "zur Familie» ihres Dienstherrn gehören, daß also eine über den wirtschaftlichen Bereich hinausgehende Bindung besteht. Dafür spricht etwa, daß die Kolta sich rasieren lassen und Trauer beobachten, wenn die Familie ihres Herrn von einem Todesfall betroffen wurde.

Hat ein Dienstherr keine Verwendung für alle Mitglieder seiner Kolta-Familie, so mag er einigen nahelegen, sich einen neuen Herrn zu suchen. Verkaufen kann er sie nicht: von Sklaverei kann also nicht gesprochen werden.

Stirbt der alleinige Ernährer einer Kolta-Familie, so hat der Dienstherr für die Hinterbliebenen zu sorgen. Uber seine Aufwendungen kann er aber Buch führen, und wenn er die Kinder des Verstorbenen aufgezogen hat, kann er von ihnen Kompensation in Form von Arbeit fordern. Heiratet eine Witwe wieder, so hat der Herr ihres neuen Gatten demjenigen ihres alten eine Entschädigung für seine Aufwendungen zu bezahlen.

Auf Gemeindeebene erscheinen die Kolta ebenfalls als Dienstleute der hohen Kasten. Dies zeigt sich etwa bei Anlaß des Diwali-Festes, das in JaunsarBawar zu Beginn des Monates Mangsir (Mitte November) abgehalten wird. Im Verlauf dieses Festes haben die Kolta nämlich den Dorfobmann auf einem Gerüst, das einen Elefanten darstellt, herumzutragen (möglicherweise geht es dabei um die Krönungszene aus dem Leben des Helden Rama), und schließlich haben sie von Haus zu Haus $\mathrm{zu}$ ziehen und den Leuten hoher Kaste Segenssprüche vorzutragen.

Hin und wieder scheint eine Kolta-Gruppe einem Dorf über den Kopf gewachsen zu sein. So bildeten sich in dem zu Dasau gehörigen Weiler Matar und in dem zu Haja gerechneten Kaletha Kolta-Kolonien, die allmählich die Form von Dörfern annahmen und weitgehende Selbständigkeit erlangten. Die Kolta von Matar wurden von ihren früheren Herren nur noch zum Grasschneiden und bei Anlaß des 
Diwali-Festes zur Teilnahme an der Elefantenzeremonie herbeigerufen.

Abweichungen vom Berufsschema sind nicht häufig. Immerhin fand ich in Dasau unter 65 über 15jährigen Rajput einen, der sich zur Erntezeit als Zwischenhändler betätigte, einen, der bei diesem als Gehilfe arbeitete, einen, der im Dienste der Forstbehörde stand, einen, der sich, ohne dabei seinen Kastenstatus einzubüßen, als Zimmermann betätigte, einen, der bei der Hebamme, die vom Sanitätsdepartement nach Dasau gesandt worden war, als Diener arbeitete und zwei, die eine höhere Schule besuchten. Dazu stellte ich fest, daß von den zwölf Bajgi des Ortes zwei für den Dorfobmann, der ihnen ein Darlehen gewährt hatte, Knechtedienste verrichteten; der eine betätigte sich zur Erntezeit als Maultiertreiber, der andere wurde wie ein Kolta zu Landarbeiten herangezogen. - In den meisten Fällen handelte es sich bei diesen Leuten um jüngste Söhne, die im väterlichen Betrieb überzählig waren oder die doch leicht entbehrt werden konnten.

$\pi$

\section{Moderne Tendenzen}

Die wirtschaftlichen Verhältnisse der niederen Kasten hatten schon 1939 einer englischen Studiengruppe mißfallen. Ihre Empfehlungen lauteten, den Kolta, Bajgi usw. sollte ermöglicht werden, Land zu besitzen, in den lokalen Räten mitzureden und sich ihrer Schulden zu entledigen (vgl. Majumdar 1922: 14 ff.). Aber erst in neuester Zeit wurden Schritte in dieser Richtung getan. Zur Zeit meines Aufenthaltes bemühten sich die Distriktsbehörden und die seit 1953 im Rahmen des "Community Development Project» tätigen Sozialarbeiter vor allem um folgendes:

1. Die Einschulung der Kinder: In der Zeit zwischen 1947 und 1950 hatten die Distriktsbehörden die Zahl der Dorfschulen von 21 auf 94 erhöht. Es zeigte sich aber bald, daß die Kolta-Kinder den Schulen fernblieben: die hohen Kasten sahen für sich keinen Nutzen in der Ausbildung dieser Kinder, und die Kolta hielten sich für zu gering, um den hohen Kasten die Stirn zu bieten. Fortschrittliche Lehrer und Sozialarbeiter kämpften indessen gegen die Widerstände. So durfte denn auch in Dasau schließlich einer von den drei im richtigen Alter stehenden Kolta-Knaben in die Schule eintreten. Seine Familie hoffte, er werde eines Tages Beamter im Tiefland. Der Vater, von einem gewissen Ehrgeiz gepackt, stellte ihn mir bezeichnenderweise mit dem eigentlich den Rajput vorbehaltenen Nachnamen Singh vor.

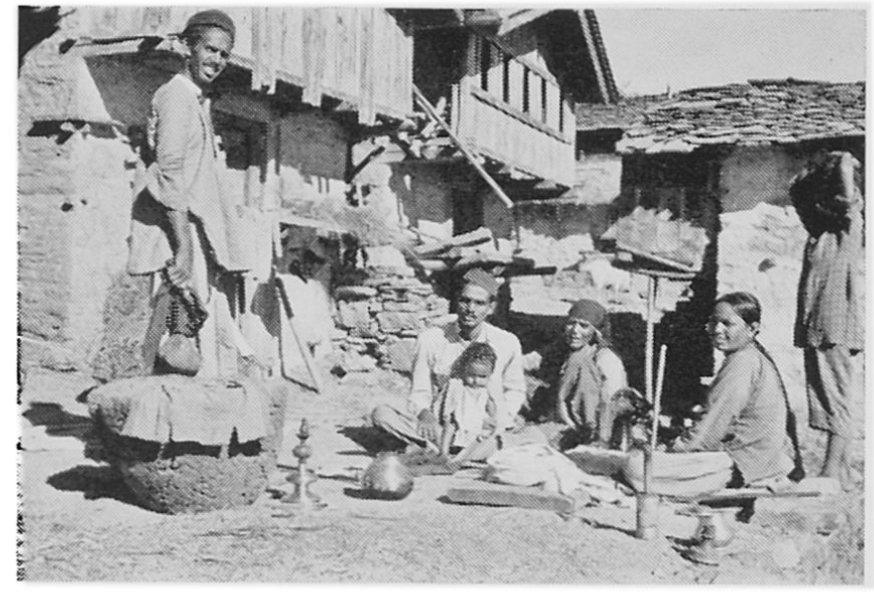

Abbildung 2. Bajgi-Familie: zwei Brüder mit Kind, Mutter und gemeinsamer Gattin (Polyandrie)

2. Die Übertragung von Landbesitz: Bei der Neueinschätzung des steuerbaren Besitzes forderten Grundbuch- und Steuerbeamte die mittleren Kasten und die Kolta auf, Land, das sie während mindestens sieben Jahren genutzt hatten, nach Bezahlung einer Entschädigung in der Höhe von zehn Jahreszinsen an den bisherigen Besitzer, auf ihren Namen eintragen zu lassen. Auch diese Aktion zeitigte aber vorerst nur bescheidenen Erfolg. Ein Kolta, den ich fragte, weshalb er der Aufforderung nicht Folge leistete, erklärte mir: "Wenn ich das bisher genutzte Land auf meinen Namen registrieren ließe, würde mein Herr zornig. Ich bin aber auf seine Gunst angewiesen, denn von dem wenigen Land könnte meine Familie nicht leben.»

3. Die Reform des Rats- und Gerichtswesens: 1953 trat auch in Jaunsar-Bawar das 1947 formulierte, als "U. P. Panchayat Raj Act» bekannte Gesetz in Kraft, das eine weitgehende Selbstverwaltung nach demokratischem Muster ermöglicht. In der Folge wurden unter behördlicher Aufsicht überall Räte und Gerichte gebildet, in denen auch die mittleren Kasten und die Kolta über Sitze verfügen. Soweit ich beobachten konnte, vermochten die Vertreter der untern Kasten in ihnen allerdings noch keine wesentliche Rolle zu spielen.

Zur Zeit meines Aufenthaltes war die Macht der hohen Kasten aber doch nicht mehr ungebrochen. Ein Kolta von Kaletha hatte den Brahmanen von Haja den Dienst gekündigt, indem er an Diwali nicht mehr geholfen hatte, den Dorfobmann auf dem «Elefanten» herumzutragen (s. oben). Die Brahmanen hatten zur Strafe seine Schafe getötet, waren aber vom Bezirksgericht verurteilt worden, Schadenersatz zu leisten. - Die Badhi von Haja hatten beschlossen, dem Dorfobmann die Herzen 
der Ziegen, die sie zu Beginn des Festmonates Magh (Mitte Januar) schlachteten, nicht mehr abzuliefern. - In Dasau, schließlich, erzählte mir einmal ein Rajput, ein Kolta, der ihm 200 Rupien schulde, sei ihm entlaufen. Als ich ihn fragte, was er nun zu tun gedenke, antwortete er resigniert: "Nichts, denn die Kolta sind heute stärker als wir.»

\section{$\operatorname{Schlu} \beta$}

Vergleicht man das Kastensystem der Jaunsari mit Kastensystemen aus dem indischen Tiefland, so findet man einerseits grundlegende Ähnlichkeiten, anderseits aber auch Unterschiede. Greifen wir die auffälligsten heraus:

Wie im Tiefland nehmen auch in Jaunsar-Bawar die Brahmanen oder "Priester» den höchsten und die Kshatriya (Rajput) oder «Krieger» den zweithöchsten Platz in der sozialen Stratifikation ein. Die dritte hinduistische Hauptkaste, die der Vaishya (Händler und Bauern), fehlt in Jaunsar-Bawar. Die übrigen jaunsarischen Kasten entsprechen aber wieder einigermaßen den professionellen Kasten des Tieflandes, nur ist ihre Zahl verhältnismäßig gering. - Wie bei den Hindu des Tieflandes bildet der Begriff einer religiös-rituellen Reinheit bei den Jaunsari die Grundlage vieler Kastenvorschriften; dagegen spielt der Begriff des Verdienstes (Karma) praktisch keine Rolle. - Wie bei den Hindu des Tieflandes engen auch bei den Jaunsari Endogamievorschriften die Wahl der Ehepartner ein; aber während im Tiefland allen Kasten hypogame Zwischenheiraten streng verboten sind, dulden die Jaunsari Eheschließungen zwischen gewissen Brahmanen und Rajput und zwischen mittleren Kasten. Auch im Tiefland werden die niederen Kasten wirtschaftlich von den hohen beherrscht; indessen stellt die Beziehung der Kolta zu den jaunsarischen Landbesitzern doch einen Spezialfall dar.

Fern vom Hauptstrom des brahmanischen Denkens hat sich in Jaunsar-Bawar offensichtlich eine verhältnismäßig grobe Version des hinduistischen Kastensystems entwickelt.

\section{Literatur}

Haas, S. 1965. Die "Polyandrie» der Jaunsari (Nordindien). Anthropos., vol. 60, p. 369-386. Madan, T. N. 1965. Family and Kinship, a Study of the Pandits of Rural Kashmir. London.

Majumdar, D. N. 1962. Himalayan Polyandry. Bombay.

Saksena, R. N. 1955. Social Economy of a Polyandrous People. Agra.

\section{Anmerkungen}

1 und 2. Die Grundbedeutung der Namen Kolta (Koi, Koli) und Hali war meinen Informanten nicht bekannt. Ich möchte aber darauf hinweisen, daß $\mathrm{Kol}$ und $\mathrm{Har}$ in den Munda-Sprachen "Mensch» bedeutet (vgl. Encyclopaedia Britannica 1960, Bd. 13).

3. Von Dewar-Kindern erhielt ich das folgende Vokabular:

\section{Kirkoali Mehl}

Kirkoali tangenge? Nehmt ihr Mehl?

Kheda (d zerebral) Branntwein

Chinkra Ziegenbock

Chilka Öl

Nanguli Hirse

Matkiyal Reis

Remda ( $d$ zerebral) Widder

Remdi ( $d$ zerebral) Schaf

Roit Hülsenfrüchte

Lumbil Schmelzbutter

Loike Weizen

Jamriya Brahmane

Kocchiya Rajput

Khuloi Badhi, Sonar

Zingiya Daki-Bajgi

Goiyan Kolta

\section{Summary}

The author describes the caste system of the Jaunsari, a Pahari people living in the Subhimalayan mountains of Jaunsar-Bawar (U. P., Northern India). A supplementary note (Anm. 3) contains a short vocabulary of the secret language used by the members of the Dewar caste (a caste of temple servants). 